\begin{tabular}{|c|c|}
\hline Title & Filament formation and erasure in molybdenum oxide during resistive switching cycles \\
\hline Author(s) & Kudo, Masaki; A rita, Masashi; Ohno, Y uuki; Takahashi, Y asuo \\
\hline Citation & $\begin{array}{l}\text { A pplied Phy sics Letters, 105(17), } 173504 \\
\text { https://doi.org/10.1063/1.4898773 }\end{array}$ \\
\hline Issue Date & $2014-10-27$ \\
\hline Doc URL & http:/hdl.handle.net/2115/57674 \\
\hline Rights & $\begin{array}{l}\text { Copyright } 2014 \text { A merican Institute of Physics. This article may be downloaded for personal use only. A ny other use } \\
\text { requires prior permission of the author and the A merican Institute of Physics. The following article appeared in A ppl. } \\
\text { Phys. Lett. 105, 173504(2014) and may be found at } \\
\text { http://scitation.aip.org/content/aip/ ournal/apl/105/17/10.1063/1.4898773 }\end{array}$ \\
\hline Tyре & article \\
\hline File Information & 1.4898773.pdf \\
\hline
\end{tabular}

Instructions for use 


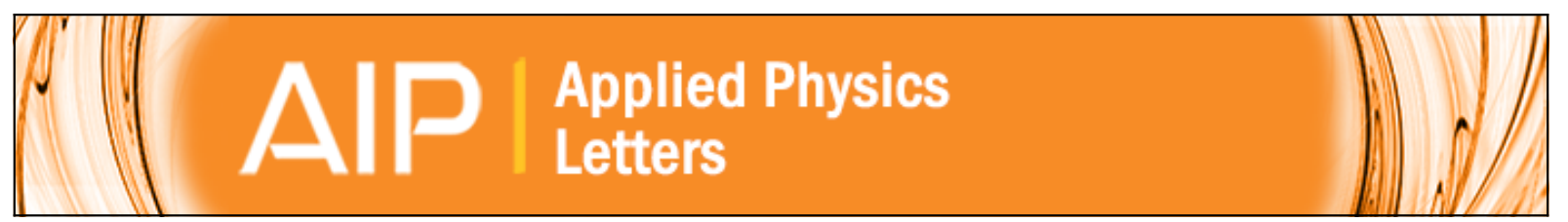

Filament formation and erasure in molybdenum oxide during resistive switching cycles

Masaki Kudo, Masashi Arita, Yuuki Ohno, and Yasuo Takahashi

Citation: Applied Physics Letters 105, 173504 (2014); doi: 10.1063/1.4898773

View online: http://dx.doi.org/10.1063/1.4898773

View Table of Contents: http://scitation.aip.org/content/aip/journal/apl/105/17?ver=pdfcov

Published by the AIP Publishing

\section{Articles you may be interested in}

Observation of bias-dependent noise sources in a TiOx/TiOy bipolar resistive switching frame

Appl. Phys. Lett. 104, 083508 (2014); 10.1063/1.4865783

Impact of Joule heating on the microstructure of nanoscale $\mathrm{TiO} 2$ resistive switching devices

J. Appl. Phys. 113, 163703 (2013); 10.1063/1.4803033

Influence of molybdenum doping on the switching characteristic in silicon oxide-based resistive switching memory

Appl. Phys. Lett. 102, 043508 (2013); 10.1063/1.4790277

Semiconducting-like filament formation in TiN/HfO2/TiN resistive switching random access memories Appl. Phys. Lett. 100, 142102 (2012); 10.1063/1.3696672

Impact of $\mathrm{TaOx}$ nanolayer at the GeSex/W interface on resistive switching memory performance and investigation of $\mathrm{Cu}$ nanofilament

J. Appl. Phys. 111, 063710 (2012); 10.1063/1.3696972

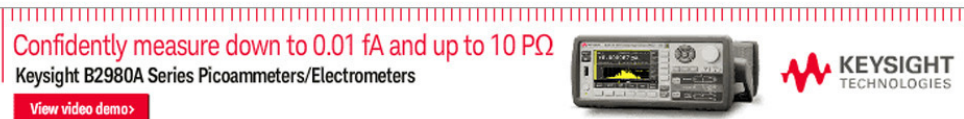




\title{
Filament formation and erasure in molybdenum oxide during resistive switching cycles
}

\author{
Masaki Kudo, Masashi Arita, ${ }^{\text {a) }}$ Yuuki Ohno, and Yasuo Takahashi \\ Graduate School of Information Science and Technology, Hokkaido University, Sapporo 060-0814, Japan
}

(Received 2 September 2014; accepted 6 October 2014; published online 27 October 2014)

\begin{abstract}
In-situ filament observations were carried out on the $\mathrm{Cu} / \mathrm{MoO}_{\mathrm{x}} / \mathrm{TiN}$ resistive random access memory (ReRAM) by using transmission electron microscopy. Multiple positive and negative $I-V$ cycles were investigated. Clear set-reset bipolar switch corresponding to the characteristics of conventional ReRAM devices was recognized. Filament grew from TiN to $\mathrm{Cu}$ in the set cycle and shrank from TiN to $\mathrm{Cu}$ in the reset cycle. However, there was no clear contrast change in the image at the switching moment, and thus, switching is thought to occur in a local region of the filament. When the current was large at reset, the filament shrank much, and its position tended to change. (C) 2014 AIP Publishing LLC. [http://dx.doi.org/10.1063/1.4898773]
\end{abstract}

Resistive random access memory (ReRAM) has attracted much attention as a next-generation nonvolatile memory. ${ }^{1-6}$ Its advantages include high-speed operation, low power consumption, and high integration. By applying voltage, the resistance changes between high and low resistance states (HRS and LRS). The current-voltage ( $I-V)$ curve exhibits hysteresis that can be exploited in nonvolatile ReRAMs. Since an early report of a Perovskite-type oxide, ${ }^{7}$ various materials including binary oxides ${ }^{8-13}$ and solid electrolytes $^{14-24}$ have been reported on this switching operation.

The pristine state is typically HRS. It converts into LRS by applying voltage in the "forming" and "set" processes. Subsequent voltage application returns the resistance into HRS in a "reset" process. Reproducible resistive switching between HRS and LRS is achieved by repeating these processes. There are two switching types: unipolar and bipolar. While the same voltage polarity is used in unipolar switching, polarity alternation is required in bipolar switching. In many cases, formation and rupture of the filament is discussed. ReRAM with a solid electrolyte sandwiched between an active top electrode (TE, e.g., $\mathrm{Cu}$ ) and an inert bottom electrode (BE, e.g., Pt) shows bipolar switching. Its operation is expected as follows. ${ }^{2,5,19,22,23}$ Positive voltage to the TE oxidizes $\mathrm{Cu}$ into cations. The $\mathrm{Cu}$ ions move along the electric field toward the BE, are metallized there, and grow toward the TE. When this conductive filament bridges the $\mathrm{BE}$ and the TE, the resistance becomes low (set operation). The opposite reaction occurs after voltage reversal, and the filament is ruptured (reset operation). However, detailed behavior of the filament on the switching operation is still obscure.

To investigate the switching operation in real space, in-situ transmission electron microscopy (TEM) $)^{25-30}$ has been applied to ReRAMs of various solid electrolytes. ${ }^{31-34}$ Fujii et al. ${ }^{31,32}$ dynamically observed the growth and rupture of a conductive filament made of $\mathrm{Cu}$ in the $\mathrm{Cu}-\mathrm{Ge}-\mathrm{S}$ layer deposited on a needle-shaped Pt-Ir electrode. This filament grew from the cathode and shrank to the same electrode after

\footnotetext{
${ }^{\text {a) }}$ Author to whom correspondence should be addressed. Electronic mail: arita@nano.ist.hokudai.ac.jp.
}

polarity change of the voltage. Yang et al. ${ }^{33}$ used some materials as the switching layer and discussed the effect of cation mobility on filament formation. Although in-situ TEM has provided important information, the sample was specialized for TEM, the operation was quite slow, and the current was much less than a few $\mu \mathrm{A}$. To gain insight into realistic ReRAM devices, dynamical structural changes should be simultaneously observed during multiple switching cycles.

In this work, in-situ TEM observations were carried out on $\mathrm{Cu} / \mathrm{MoO}_{\mathrm{x}} / \mathrm{TiN}$ ReRAM. Here, $\mathrm{Cu}$ was an active TE, $\mathrm{MoO}_{\mathrm{x}}$ was a switching layer, ${ }^{17,21}$ and TiN was an inert BE. Multiple bipolar $I-V$ cycles were realized during the TEM observation. The $\mathrm{Cu}$-based filament grew from $\mathrm{TiN}$ to $\mathrm{Cu}$ in the set cycle (current from $\mathrm{Cu}$ to $\mathrm{TiN}$ ) and became thicker with the compliance current. The filament ruptured in the reset cycle. However, at the switching moment, there was no clear change in the TEM image contrast. This indicates that the ReRAM switching must occur in a local region of the filament. With a large current from TiN to $\mathrm{Cu}$, the filament completely disappeared, and its position then changed.

The sample structure was $\mathrm{Pt}_{(100 \mathrm{~nm})} / \mathrm{Cu}_{(30 \mathrm{~nm})} / \mathrm{MoO}_{\mathrm{x}}(50 \mathrm{~nm})$ on the TiN (BE)/Si substrate. First, the substrate was treated with oxygen plasma to remove polymer residual covering the substrate surface. X-ray photoelectron spectroscopy showed the oxidized TiN surface. The $\mathrm{MoO}_{\mathrm{x}}$ layer was prepared by reactive RF sputtering of Mo with $\mathrm{Ar}_{-} \mathrm{O}_{2}$ (20\%). The Mo 3d peak positions were those of $\mathrm{MoO}_{3}$. No Bragg peaks were evident in the $\mathrm{x}$-ray diffraction pattern, indicating the amorphous nature of the film. Next, without breaking the vacuum, the $\mathrm{Cu}$ TE and Pt cap layers were deposited by conventional RF sputtering. TEM samples were prepared using the ion-shadow method, ${ }^{35,36}$ which is an ion milling technique with carbon mask particles. Many cone-shaped Si needles containing miniaturized ReRAM devices were formed on top. In-situ TEM observations were performed using a JEM-2010 microscope $\left(200 \mathrm{kV}, 10^{-5} \mathrm{~Pa}\right)$. The sample was placed in a home-made TEM/STM holder. ${ }^{28}$ It was fixed, while the Pt-Ir probe was movable. For electrical measurements, a Yokogawa GS820 source-measure-unit was used. After connecting the probe to the Pt layer, the bias voltage was applied to $\mathrm{Pt} / \mathrm{Cu}$, while the substrate was grounded. Typically, the voltage was swept 
between about $\pm 3.0 \mathrm{~V}$ at a rate of $0.7-0.8 \mathrm{Vs}^{-1}$. Image dynamics was recorded with a CCD camera $\left(30\right.$ frames s $\left.^{-1}\right)$.

An example of the $I-V$ curve during the second switching cycle is shown in Fig. 1(a). The switching layer was nearly a disk of $\phi 350 \mathrm{~nm}$. The compliance current was $400 \mu \mathrm{A}$. By increasing the positive voltage (Cu TE biased) from states A to B in Fig. 1(a), the current increased gradually and then abruptly jumped to the compliance value at $2.6 \mathrm{~V}$ (B to C). This is the set operation to LRS. After changing the positive voltage (D and $\mathrm{E}$ ), it was reduced to zero (F). Waiting $5 \mathrm{~min}$, the polarity was reversed $\left(\mathrm{F}^{\prime}\right)$, and the resistance was LRS. Between states $\mathrm{G}$ and $\mathrm{H}$, the current exhibited two small jumps. These were the reset switching to HRS. The negative current reached to $-400 \mu \mathrm{A}$ with increasing the negative voltage to $-3 \mathrm{~V}$ ( $\mathrm{I}$ and $\mathrm{J}$ ), and a clear hysteresis character was recognized. Finally, the voltage was set to zero (K). This cycle denotes bipolar switching. The resistance ratio of HRS/LRS was obtained from this graph to be 68 at $+0.5 \mathrm{~V}$ and 17 at $-0.5 \mathrm{~V}$. The estimated resistance values include the resistance of the $\mathrm{Si}$ substrate connected in series to the ReRAM, and thus, the intrinsic resistance ratio must be larger than these values.

To clarify that the result in Fig. 1(a) is not a special case limited to in-situ TEM measurements, we compared it with data from an ex-situ measurement of a device having a conventional geometry (Fig. 1(b)). The stacking sequence and the layer thicknesses were the same as those of the TEM samples, while the switching area was large $(\phi 16 \mu \mathrm{m})$. Figure 1(b) shows a clear example of bipolar switching. Abrupt set switching was realized at about $1.4 \mathrm{~V}$, and gradual reset switching started at about $-0.8 \mathrm{~V}$. The property of these two $I$-V curves (Figs. 1(a) and 1(b)) agrees well. It can be concluded that the vacuum environment and electron beam irradiation did not have any negative effect.

The TEM video corresponding to Fig. 1(a) is shown in Fig. 2. The $\mathrm{MoO}_{\mathrm{x}}$ layer with bright contrast is sandwiched between two dark regions of $\mathrm{Pt} / \mathrm{Cu}$ and TiN/Si. The overall switching area was observable. The resistance before starting the cycle was HRS. This set/reset movie shows that a filament-like dark contrast appeared in the set process and disappeared in the reset process, indicating that this dark filament behaved as the conductive filament. The filament was about $35 \mathrm{~nm}$ wide. It can be recognized in the video that part of the $\mathrm{Cu}$ layer suddenly became brighter at the set switching. This indicates quick movement of $\mathrm{Cu}$ to form the filament. In addition, we analyzed the filament (but in another
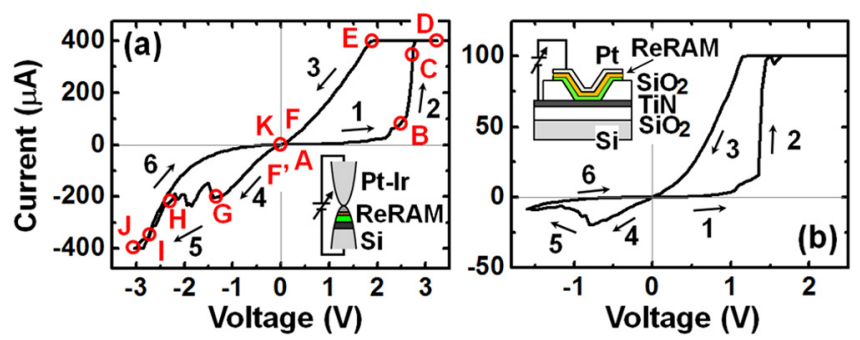

FIG. 1. $I$ - $V$ characteristics of (a) the TEM sample during in-situ observation and (b) an conventional ReRAM device. Insets are schematics of the samples; (a) $\phi 350 \mathrm{~nm}$ and (b) $\phi 16 \mu \mathrm{m}$. Arrows with numbers indicate the sweeping direction. Alphabetical symbols correspond to images in Fig. 3.

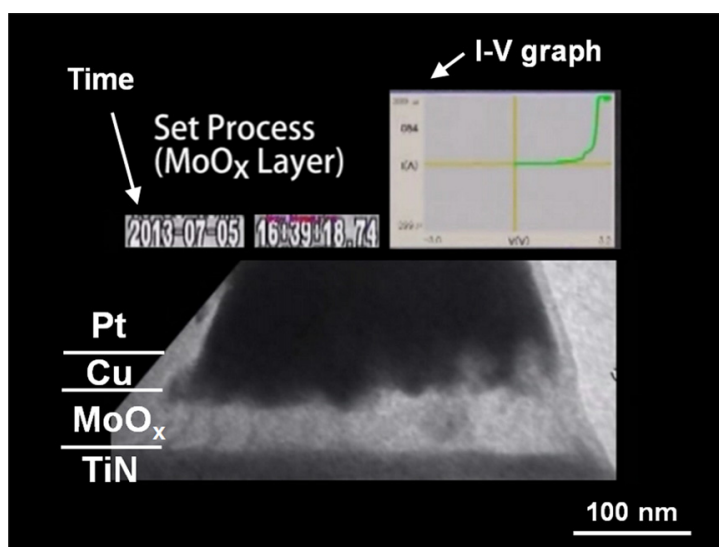

FIG. 2. Video movie of a ReRAM operation cycle with the frames of the $I-V$ graph and time. The left part of the image was darker than the right part. This was caused by imperfection of the experimental condition. The center of the electron beam for the TEM observation was slightly shifted to the upper right. (Multimedia view) [URL: http://dx.doi.org/10.1063/1.4898773.1].

sample) by using energy dispersive $\mathrm{x}$-ray spectroscopy and found that it contained more $\mathrm{Cu}$ than the other regions in the $\mathrm{MoO}_{\mathrm{x}}$ layer. We conclude that the filament is made up largely of $\mathrm{Cu}$, and the ReRAM switching occurs with the appearance and disappearance of this filament.

To discuss in detail, filament images were extracted from the video (Figs. 3 and 4). Symbols A to K in Figs. 3(a) and 4(a) correspond to the states in Fig. 1(a). Image $\mathrm{F}^{\prime}$ in Fig. 4(a) was taken 5 min after image F in Fig. 3(a). At first, we consider the set process shown in Fig. 3. Between states $\mathrm{A}$ and $\mathrm{B}$, the resistance was HRS without any drastic image change. However, at an abrupt set switching, a small dark contrast appeared near the TiN BE. This contrast, which seems to be a nucleus of the filament, grew at state $\mathrm{C}$ (frame number of \#100). Details around state C (\#100) are provided in Fig. 3(b), where five images are shown in sequence at 30 ms intervals. While the current almost reached the compliance value $(400 \mu \mathrm{A})$ at $\mathrm{C}$, the dark filament did not connect to the $\mathrm{Cu}$ TE. The current may be ionic contributed by movement of dense $\mathrm{Cu}$ ions at the gap between the filament and the $\mathrm{Cu}$ TE because the resistance was low. Until state D

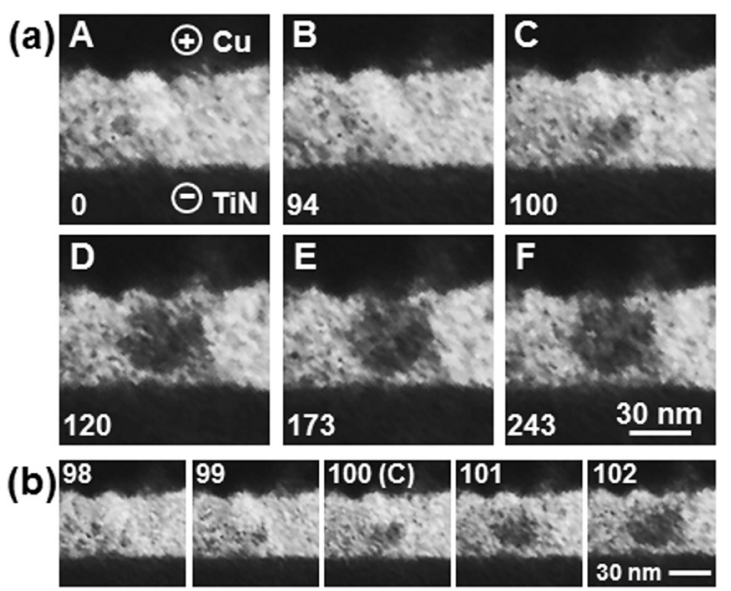

FIG. 3. Magnified filament images in the set process, which were extracted from the video. Numbers indicate the order of video frames counted from the image A. The interval of the video frames was $30 \mathrm{~ms}$. (a) Images A-F corresponding to the states in Fig. 1(a). (b) Details of the filament evolution around $\mathrm{C}$. 
(a) $\mathrm{F}$
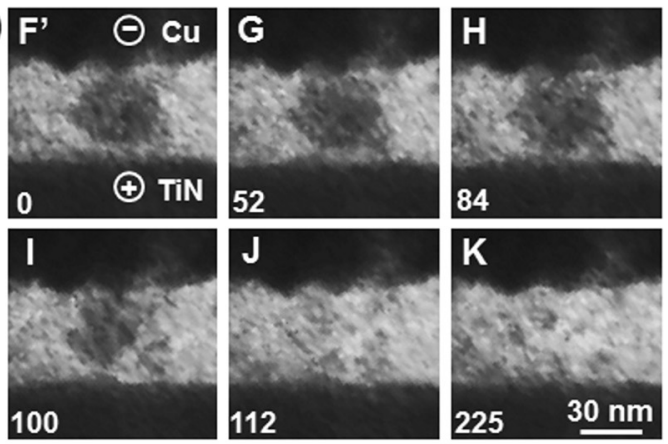

(b) 8
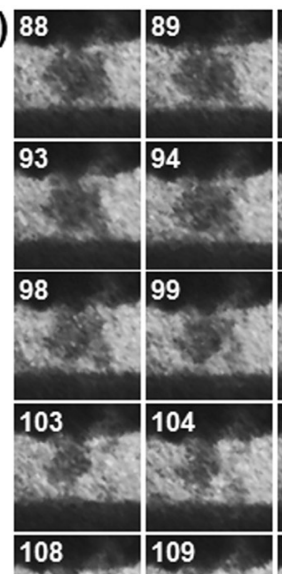

108 94
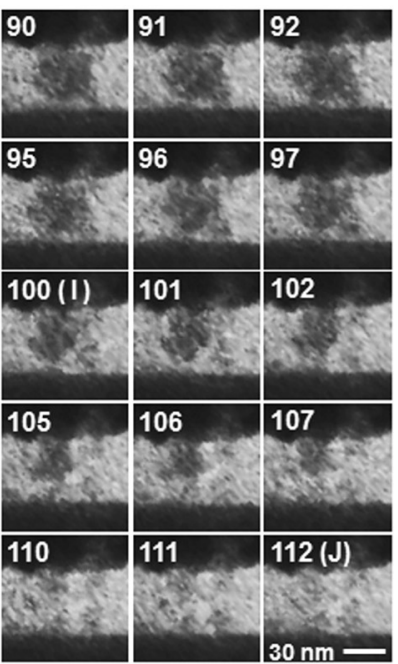

FIG. 4. Magnified filament images in the reset process, which were extracted from the video. Numbers indicate the order of video frames counted from the image $\mathrm{F}^{\prime}$. The interval of the video frames was $30 \mathrm{~ms}$. (a) Images $\mathrm{F}^{\prime}-\mathrm{K}$ corresponding to the states in Fig. 1(a). Image $\mathrm{F}^{\prime}$ was taken 5 min after image $\mathrm{F}$ in Fig. 3(a). (b) Detailed sequence of filament shrinkage around H, I, and J. By continuing large negative current, the filament shrank to the $\mathrm{Cu}$ TE.

(\#120) at $600 \mathrm{~ms}$ (20 frames) after C, the filament grew towards the $\mathrm{Cu}$ TE. At the same time, it became thick to be about $35 \mathrm{~nm}$ in width. After the nucleus appeared, the filament bridged two electrodes within about $200 \mathrm{~ms}$ ( 7 frames after $\mathrm{C}$; the image is not shown here). When the voltage was reduced to zero ( $\mathrm{E}$ and $\mathrm{F})$, the filament image was unchanged, and the resistance was LRS. Summarizing the result in Fig. 3, a nucleus of the conductive filament appeared near the TiN electrode and grew along the $\mathrm{Cu}$ electrode at the set operation. This fits well with the electrochemical switching model. ${ }^{2,5,25,30,31}$ Based on Refs. 33 and 34, the $\mathrm{Cu}$ ion mobility is high in this case. In order to simulate real ReRAM switching with quick response, ${ }^{15,16,21}$ we introduced a compliance current of the order of $10^{2} \mu \mathrm{A}$, while many other in-situ TEM reports used a current of less than a few $\mu \mathrm{A}$. The Joule heat seems to be what increases the ion mobility.

As shown in Fig. 1, the resistance change in the reset process is gradual compared to that in the set process. This is recognizable also in previous reports. The sequential images in the reset process are shown in Fig. 4(a). The resistance was LRS between states $F^{\prime}$ and G. Even after the two-step weak reset, image $H$ (frame number of \#84) had a similar contrast to $\mathrm{F}^{\prime}$ and $\mathrm{G}$ while the resistance increased. This suggests that the reset switching occurred very locally in the filament, probably at singular positions such as the ends of filaments touching the electrodes. ${ }^{2,5,19,22,23}$ When we further increased the negative voltage to $-2.6 \mathrm{~V}$, the negative current increased beyond $-300 \mu \mathrm{A}$ (image I, \#100). The filament then shrank toward the $\mathrm{Cu}$ TE. By carefully checking the video (Fig. 2), it looked to be sucked up to the $\mathrm{Cu}$ layer. We occasionally observed this phenomenon also in other samples. When the current reached the compliance value, the filament vanished, although some residuals remained (image J, \#112). The details around H, I, and J are clearly recognizable in Fig. 4(b) of frames between \#88 and \#112. The filament destruction observed here does not fit with reported models. ${ }^{19,22,23}$ As described above, the TiN surface was oxidized. This region must have higher resistance than the other region in the filament, and the generated Joule heat is concentrated here. This is possible why the filament was first broken near the interface with TiN (anode with this voltage polarity). Even with this speculation, the shrinkage toward the $\mathrm{Cu}$ TE (cathode) is hard to be explained only with considering a metallic $\mathrm{Cu}$ filament. Larentis et al. ${ }^{37}$ considered a conductive filament of dense cations and/or oxygen vacancies and reproduced a gradual reset by combining thermal and electric field effects. Shrinkage occurred from the anode to the cathode. Although their precondition does not exactly fit with our work, the results may confirm some relationship. Regardless of the physical and chemical model of ReRAM switching, the filament shrinkage in this work was affected by Joule heat, since it occurred at a relatively high current.

Here, we discuss the filament size and position. In this series of measurements, five set/reset switching cycles were investigated. The compliance current $\left(I_{\text {comp }}\right)$ was stepwise increased to $1 \mathrm{~mA}$, at which point the device was destructed. The filament was completely erased in the reset procedure. Figure 5 shows the TEM images taken just after the set
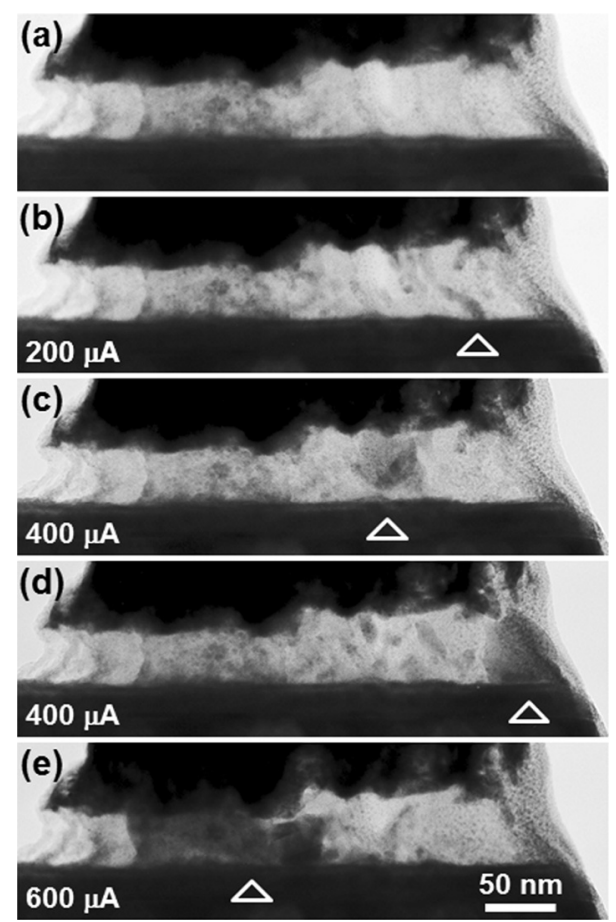

FIG. 5. TEM images of the ReRAM in the (a) initial state and (b)-(e) LRS after the continuous set operations with increasing compliance current as indicated in each figure. Triangles are the filament positions. 
process. The initial state is seen in Fig. 5(a). The sample was HRS, and the contrast in this image had no relation to the ReRAM switching. The filament in the first set with $I_{\text {comp }}=200 \mu \mathrm{A}$ was thin, with a width of $7 \mathrm{~nm}$ (Fig. 5(b)). The filament widths were 35,45 , and $100 \mathrm{~nm}$ for 400,400 , and $600 \mu \mathrm{A}$, respectively (Figs. 5(c)-5(e)). As expected earlier, ${ }^{16,22}$ the filament was thicker with $I_{\text {comp. Its position }}$ moved along the left in the second set (Fig. 5(c)) and changed to the right in the third set (Fig. 5(d)). In the fourth set, it moved to the left (Fig. 5(e)). This movement does not fit with the conventional model without changing the filament position. Fujii et $a l .^{32}$ reported that the filament appears at the same position even after erasure and suggested the existence of residual filament nuclei. Based on this, we assume that nuclei were removed after the complete filament erasure (over-reset). Gradual reset is desirable for multilevel analog memories. ${ }^{37}$ However, over-reset induces a position change of the filament resulting in switching instability.

In summary, we observed the real-time formation and rupture of the conductive filament inside $\mathrm{Cu} / \mathrm{MoO}_{\mathrm{x}} / \mathrm{TiN}$. In the set process, the filament grew from the $\mathrm{BE}(\mathrm{TiN})$ toward the TE $(\mathrm{Cu})$. However, it did not connect with the TE at the switching moment. It became thick with additional voltage application, showing a good retention property $(>5 \mathrm{~min})$ at least for TEM observations. The filament size was controllable with the current compliance. In the moment of reset to HRS, no drastic change was observed in the filament: only a tiny area seems to contribute to the filament disconnection. When the current from the BE to the TE was increased, the filament disappeared and the resistance became larger. The shrinkage direction of the filament was from the BE to the TE, which cannot be explained by the standard filament model based on a redox reaction. Although the details are not yet fully clear, there must be a strong influence from the Joule heat of the current.

This work was financially supported by KAKENHI, by the Ministry of Education, Culture, Sports, Science and Technology (MEXT) Japan, and by the Japan Society of the Promotion of Science (JSPS) (Grant Nos. 24360128, 25420279, and 26630141). Part of this work was supported by the Nanotechnology Platform Program (Hokkaido University) organized by MEXT. We are thankful to Dr. T. Tesfamichael (Queensland University of Technology) for critical reading of the manuscript.

${ }^{1}$ M. N. Kozicki, M. Park, and M. Mitkova, IEEE Trans. Nanotechnol. 4, 331 (2005).

${ }^{2}$ R. Waser and M. Aono, Nat. Mat. 6, 833 (2007).

${ }^{3}$ A. Sawa, Mater. Today 11, 28 (2008).

${ }^{4}$ H. Akinaga and H. Shima, Proc. IEEE 98, 2237 (2010).

${ }^{5}$ A. Chen, Ionic Memory Technology, in Solid State Electrochemistry II: Electrodes, Interfaces and Ceramic Membranes, 1st ed., edited by V. V. Kharton (Wiley-VCH, Weinheim, 2011).

${ }^{6}$ K. M. Kim and C. S. Hwang, Nanotechnol. 22, 254002 (2011).

${ }^{7}$ S. Q. Liu, N. J. Wu, and A. Ignatiev, Appl. Phys. Lett. 76, 2749 (2000).

${ }^{8}$ S. Seo, M. M. Lee, D. H. Seo, E. J. Jeoung, D.-S. Suh, Y. S. Joung, I. K. Yoo, I. R. Hwang, S. H. Kim, I. S. Byun, J.-S. Kim, J. S. Choi, and B. H. Park, Appl. Phys. Lett. 85, 5655 (2004).
${ }^{9}$ B. J. Choi, D. S. Jeong, S. K. Kim, C. Rohde, S. Choi, J. H. Oh, H. J. Kim, C. S. Hwang, K. Szot, R. Waser, B. Reichenberg, and S. Tiedke, J. Appl. Phys. 98, 033715 (2005).

${ }^{10}$ H. Shima, F. Takano, H. Akinaga, Y. Tamai, I. H. Inoue, and H. Takagi, Appl. Phys. Lett. 91, 012901 (2007).

${ }^{11}$ S. Muraoka, K. Osano, Y. Kanzawa, S. Mitani, S. Fujii, K. Katayama, Y. Katoh, Z. Wei, T. Mikawa, K. Arita, Y. Kawashima, R. Azuma, K. Kawai, K. Shimakawa, A. Odagawa, and T. Takagi, Tech. Dig. Int. Electron Devices Meet. 2007, 779 .

${ }^{12}$ M.-J. Lee, C. B. Lee, D. Lee, S. R. Lee, M. Chang, J. H. Hur, Y.-B. Kim, C.-J. Kim, D. H. Seo, S. Seo, U-I. Chung, I.-K. Yoo, and K. Kim, Nat. Mat. 10, 625 (2011).

${ }^{13}$ M. Arita, H. Kaji, T. Fujii, and Y. Takahashi, Thin Solid Films 520, 4762 (2012).

${ }^{14}$ M. N. Kozicki, M. Yun, L. Hilt, and A. Sing, in Proceedings of Solid-State Ionic Devices: 195th Electrochemical Society Proceedings, Vol. 99-13, Seattle, USA, 1999, May 1999, edited by E. D. Wachsman, J. R. Akridge, M. Liu, and N. Yamazoe (Electrochem. Soc., Pennington, NJ, 1999), pp. 298-309.

${ }^{15}$ K. Aratani, K. Ohba, T. Mizuguchi, S. Yasuda, T. Shiimoto, T. Tsushima, T. Sone, K. Endo, A. Kouchiyama, S. Sasaki, A. Maesaka, N. Yamada, and H. Narisawa, Tech. Dig. Int. Electron Devices Meet. 2007, 783.

${ }^{16}$ U. Russo, D. Kamalanathan, D. Ielmini, A. L. Lacaita, and M. N. Kozicki, IEEE Trans. Electron Devices 56, 1040 (2009).

${ }^{17}$ D. Lee, D.-J. Seong, I. Jo, F. Xiang, R. Dong, S. Oh, and H. Hwang, Appl. Phys. Lett. 90, 122104 (2007).

${ }^{18}$ C. Schindler, M. Weides, M. N. Kozicki, and R. Waser, Appl. Phys. Lett. 92, 122910 (2008).

${ }^{19}$ N. Banno, T. Sakamoto, N. Iguchi, H. Sunamura, K. Terabe, T. Hasegawa, and M. Aono, IEEE Trans. Electron Devices 55, 3283 (2008).

${ }^{20}$ C. Kügeler, R. Rosezin, R. Weng, R. Waser, S. Menzel, B. Klopstra, and U. Böttger, in Proceedings of the 9th Nanotechnology Conference: IEEE NANO 2009, Genoa, Italy, 26-30 July 2009 (IEEE, Piscataway, 2009) pp. $1102-1105$.

${ }^{21}$ J. Yoon, H. Choi, D. Lee, J.-B. Park, J. Lee, D.-J. Seong, Y. Ju, M. Chang, S. Jung, and H. Hwang, IEEE Trans. Electron Device Lett. 30, 457 (2009).

${ }^{22}$ Y. Bernard, V. T. Renard, P. Gonon, and V. Jousseaume, Microelect. Eng. 88, 814 (2011).

${ }^{23}$ T. Tsuruoka, K. Terabe, T. Hasegawa, and M. Aono, Nanotechnol. 21, 425205 (2010).

${ }^{24}$ S. Hasegawa, K. Kinoshita, S. Tsuruta, and S. Kishida, ECS Trans. 50, 61 (2013).

${ }^{25}$ Ch. Jooss, J. Hoffmann, J. Fladerer, M. Ehrhardt, T. Beetz, L. Wu, and Y. Zhu, Phys. Rev. B 77, 132409 (2008).

${ }^{26}$ T. Fujii, H. Kaji, H. Kondo, K. Hamada, M. Arita, and Y. Takahashi, IOP Conf. Ser.: Mater. Sci. Eng. 8, 012033 (2010).

${ }^{27}$ D.-H. Kwon, K. M. Kim, J. H. Jang, J. M. Jeon, M. H. Lee, G. H. Kim, X.S. Li, G.-S. Park, B. Lee, S. Han, M. Kim, and C. S. Hwang, Nat. Nanotechnol. 5, 148 (2010).

${ }^{28}$ T. Fujii, M. Arita, K. Hamada, H. Kondo, H. Kaji, Y. Takahashi, M. Moniwa, I. Fujiwara, T. Yamaguchi, M. Aoki, Y. Maeno, T. Kobayashi, and M. Yoshimaru, J. Appl. Phys. 109, 053702 (2011).

${ }^{29}$ T. Fujii, M. Arita, K. Hamada, Y. Takahashi, and N. Sakaguchi, J. Appl. Phys. 113, 083701 (2013).

${ }^{30}$ J.-Y. Chen, C.-L. Hsin, C.-W. Huang, C.-H. Chiu, Y.-T. Huang, S.-J. Lin, W.-W. Wu, and L.-J. Chen, Nano Lett. 13, 3671 (2013).

${ }^{31}$ T. Fujii, M. Arita, Y. Takahashi, and I. Fujiwara, Appl. Phys. Lett. 98, 212104 (2011).

${ }^{32}$ T. Fujii, M. Arita, Y. Takahashi, and I. Fujiwara, J. Mater. Res. 27, 886 (2012).

${ }^{33}$ Y. Yang, P. Gao, S. Gaba, T. Chang, X. Pan, and W. Lu, Nat. Commun. 3, 732 (2012).

${ }^{34}$ Q. Liu, J. Sun, H. Lv, S. Long, K. Yin, N. Wan, Y. Li, L. Sun, and M. Liu, Adv. Mater. 24, 1844 (2012).

${ }^{35}$ M. Kudo, M. Arita, Y. Ohno, T. Fujii, K. Hamada, and Y. Takahashi, Thin Solid Films 533, 48 (2013).

${ }^{36}$ M. Kudo, Y. Ohno, K. Hamada, M. Arita, and Y. Takahashi, ECS Trans. 58, 19 (2013).

${ }^{37}$ S. Larentis, F. Nardi, S. Balatti, D. C. Gilmer, and D. Ielmini, IEEE Trans. Electron Devices 59, 2468 (2012). 